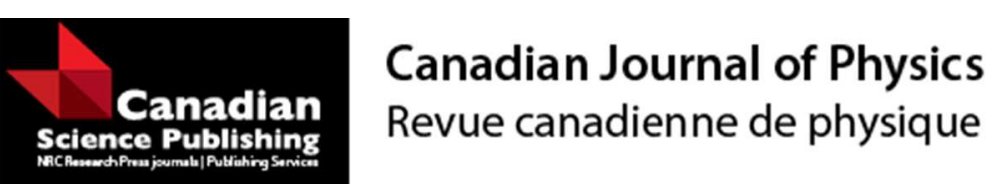

\title{
Effects of higher order corrected entropy on the black hole physics
}

\begin{tabular}{|r|l|}
\hline Journal: & Canadian Journal of Physics \\
\hline Manuscript ID & cjp-2017-0550.R1 \\
\hline Manuscript Type: & Article \\
\hline Date Submitted by the Author: & 10 -Sep-2017 \\
\hline Keyword: & $\begin{array}{l}\text { Black hole, Quantum corrections, Thermodynamics, Thermal fluctuations, } \\
\text { Statistics }\end{array}$ \\
\hline $\begin{array}{r}\text { Is the invited manuscript for } \\
\text { consideration in a Special } \\
\text { Issue? : }\end{array}$ & $\begin{array}{l}\text { Nourhassan, Behnam; Damghan University } \\
\text { Kokabi, Khodadad; Damghan university }\end{array}$ \\
\hline
\end{tabular}

\section{SCHOLARONE ${ }^{\text {m }}$ \\ Manuscripts}




\title{
Effects of higher order corrected entropy on the black hole physics
}

\author{
B. Pourhassan ${ }^{a *}$ and K. Kokabi ${ }^{a \dagger}$ \\ ${ }^{a}$ School of Physics, Damghan University, Damghan, 3671641167, Iran.
}

October 4, 2017

\begin{abstract}
In this paper we consider higher order corrections of the BTZ and AdS Schwarzschild black holes entropy and study corrected thermodynamics. We obtain some important thermodynamics quantities like internal and Helmholtz free energy and calculate specific heat. By using corrected specific heat we study black holes stability and investigate possible phase transition and critical points. Correction terms of the entropy are important when the size of black hole be small and come from thermal fluctuations of statistical physics, hence one can interpret them as quantum corrections to the black hole thermodynamics. We show that presence of corrections of entropy are many important for stability or instability of black holes.
\end{abstract}

Keywords: Black hole; Quantum corrections; Thermodynamics.

Pacs Nos: 04.70.Bw, 05.70.-a.

\section{Introduction}

Bekenstein [1] has been show that black holes satisfy standard laws of thermodynamics, which yields to the fact that the maximum of the black hole entropy is proportional to the event horizon area. It developed the holographic principle of black holes [2, 3]. Hence, generally the black hole entropy will be function of the black hole hair which are the mass, charge and angular momentum. These are the only properties which we know from every black holes so different configurations of these parameters yields to several different kinds of black holes. The black hole hairs are indeed analogous of thermodynamics variables. As we know, thermodynamics of the given system could be determined from microstates via the well known Boltzmann formula $S=k_{B} \ln \Omega(E)$, where $\Omega(E)$ is number of microstate

\footnotetext{
*Email: b.pourhassan@du.ac.ir

†Email: kokabi@du.ac.ir
} 
which obtained by differen configurations, and $k_{B}$ is Boltzmann constant. In that case, there are several works to relate black hole hair to the microstate number $[4,5,6,7,8,9$, 10]. However, it has been shown that the maximum of the black hole entropy should be corrected due to quantum fluctuations coming from the statistical mechanics, so it modifies the holographic principle $[11,12]$. These thermal fluctuations are around equilibrium of system which will be important when the size of black hole reduced due to the Hawking radiation. Hence, quantum fluctuations will correct the thermodynamics relation of the black hole by correcting the entropy. In that case there are several different ways to calculate mentioned corrections. Ref. [13] calculated the leading-order correction of the black hole entropy and found that is logarithmic. This leading-order correction recently have been used to correct thermodynamics of three-dimensional hairy black hole $[14,15,16,17]$ and shown that correction term is logarithmic at the first order approximation [18]. In that case the corrected thermodynamics and statistics of Gödel black hole due to the logarithmic correction have been studied by the Ref. [19]. The effect of leading-order thermal fluctuations on the thermodynamics of black holes in anti-de Sitter space-time has been studied by the Ref. [20]. Such quantum correction has also been used for analyzing the corrections to the thermodynamics of a black Saturn [21, 22]. Corrected thermodynamics of the modified Hayward black hole by the logarithmic term investigated by the Ref. [23]. Also, Ref. [24] have been study P-V criticality of logarithm-corrected dyonic charged AdS black holes while Ref. [25] have been study P-V criticality of AdS black holes in massive gravity. Then, thermodynamics of a small singly spinning Kerr-AdS black hole considered and effect of quantum correction investigated [26]. This logarithmic correction considered for the STU black hole and found important modification in the thermodynamics and also hydrodynamics [27]. Quantum correction of Dumb holes also calculated to examine quantum gravity effects $[28]$.

Ref. [13] applied their leading-order result to BTZ black holes, anti-de Sitter Schwarzschild and Reissner-Nordstrom black holes in arbitrary dimensions, while it is possible to consider higher order corrections [29]. In that case it is interesting to study thermodynamics of black holes with higher order corrections of the entropy. Hence, in this paper we consider BTZ and AdS Schwarzschild black holes and investigate several thermodynamics quantities under effects of higher order quantum corrections. Specially we would like to study effect of quantum corrections on the stability of black holes.

\section{Higher order corrections}

In this section we review main results obtained by the Ref. [29] to show origin of the higher order corrections of the entropy. Partition function in the canonical ensemble of $N$ particle system may given by,

$$
Z=\int_{0}^{\infty} \rho(E) e^{-\beta E} d E
$$


where $\rho(E)$ is the quantum density of the system and defined as,

$$
\rho(E)=\sum_{n} \Omega\left(E_{n}\right) \delta\left(E-E_{n}\right)
$$

where $\Omega\left(E_{n}\right)$ is number of microstates at energy $E_{n}$, while $E$ is the canonical ensemble energy average of system. We should note that the canonical partition function is the Laplace transform of the canonical density of states. Hence, one can obtain canonical density of states by Laplace inversion as,

$$
\rho(E)=\frac{1}{2 \pi i} \int_{-i \infty}^{i \infty} e^{\beta E+\ln Z} d \beta .
$$

Now, one can use Taylor expansion of the entropy around equilibrium $\beta_{0}$ and write,

$$
\begin{aligned}
S & =\beta E+\ln Z=S_{0} \\
& +\left(\frac{\partial}{\partial \beta} \ln Z\right)_{\beta_{0}}\left(\beta-\beta_{0}\right) \\
& +\frac{1}{2 !}\left(\frac{\partial^{2}}{\partial \beta^{2}} \ln Z\right)_{\beta_{0}}\left(\beta-\beta_{0}\right)^{2} \\
& +\frac{1}{3 !}\left(\frac{\partial^{3}}{\partial \beta^{3}} \ln Z\right)_{\beta_{0}}\left(\beta-\beta_{0}\right)^{3}+\cdots
\end{aligned}
$$

we know that $\left(\frac{\partial}{\partial \beta} \ln Z\right)_{\beta_{0}}=0$ because $\beta_{0}$ is an extremum, hence the second line vanishes. Therefore one can rewrite the equation (3) as follow,

$$
\rho(E)=\frac{1}{2 \pi} e^{S_{0}} \int_{-\infty}^{\infty} e^{-\frac{1}{2} \alpha_{2} y^{2}} \exp \left(\sum_{n=2}^{\infty} \frac{\alpha_{n}(i y)^{n}}{n !}\right) d y
$$

where

$$
\alpha_{n} \equiv\left(\frac{\partial^{n}}{\partial \beta^{n}} \ln Z\right)_{\beta_{0}},
$$

hence

$$
\alpha_{2} \equiv\left(\frac{\partial^{2}}{\partial \beta^{2}} \ln Z\right)_{\beta_{0}},
$$

also $\beta-\beta_{0}=i y$ change of variable is used. Then, for the large thermodynamics system one can use exponential expansion and solve integrals to find [29],

$$
\rho(E)=\frac{1}{2 \pi} e^{S_{0}} \sqrt{\frac{2 \pi}{\alpha_{2}}} \times X,
$$

where

$$
\begin{aligned}
X & \equiv 1+\sum_{n=2}^{\infty} \frac{\alpha_{2 n}(-1)^{n}}{(2 n) ! ! \alpha_{2}^{n}} \\
& +\frac{1}{2 !} \sum_{n=3}^{\infty} \sum_{m=3}^{\infty} \frac{\alpha_{n} \alpha_{m}(-1)^{k}(2 k-1) ! !}{n ! m ! \alpha_{2}^{k}}+\cdots
\end{aligned}
$$


Then, one can use $\ln (1+x) \sim x$ approximation to obtain corrected entropy as follow [29],

$$
\begin{aligned}
S & =S_{0}-\ln n-\frac{1}{2} \ln \alpha_{2} \sum_{n=2}^{\infty} \frac{\alpha_{2 n}(-1)^{n}}{(2 n) ! ! \alpha_{2}^{n}} \\
& +\frac{1}{2 !} \sum_{n=3}^{\infty} \sum_{m=3}^{\infty} \frac{\alpha_{n} \alpha_{m}(-1)^{k}(2 k-1) ! !}{n ! m ! \alpha_{2}^{k}}+\cdots
\end{aligned}
$$

We can see that leading-order correction is logarithmic while higher order corrections are also exist as a function of $S_{0}$. It should be noted that the logarithmic correction comes from thermal fluctuations while higher order correction reflects quantum effects. Ref. [29] applied above formula to the entropy of BTZ and AdS Schwarzschild black holes, and we would like to use them to study corrected thermodynamics of the mentioned black holes. Hence, in the next section we consider BTZ black hole.

\section{BTZ black hole thermodynamics}

Three dimensional BTZ black hole may be given by the following metric [30, 31],

$$
\begin{aligned}
d s^{2}= & -\left(\frac{r^{2}}{l^{2}}-8 M\right) d t^{2}+\left(\frac{r^{2}}{l^{2}}-8 M\right)^{-1} d r^{2} \\
& +r^{2} d \theta^{2}
\end{aligned}
$$

where $M$ denotes the black hole mass and $l$ is related to the negative cosmological constant via $\Lambda=-\frac{1}{l^{2}}$.

Hawking temperature of black hole is given by,

$$
T=\frac{r_{+}}{2 \pi l^{2}}
$$

where

$$
r_{+}=2 l \sqrt{2 M}
$$

is event horizon radius. Also uncorrected entropy of BTZ black hole given by,

$$
S_{0}=\frac{\pi r_{+}}{2}=\pi l \sqrt{2 M}
$$

By using the equations (13) and (14) one can obtain relation between temperature and entropy as follow,

$$
T=\frac{S_{0}}{\pi^{2} l^{2}}
$$

Hence one can obtain,

$$
S_{0}=\frac{\pi^{2} l^{2}}{\beta},
$$


where $\beta=\frac{1}{T}$ and unit value is used for the Boltzmann constant. In that case the black hole mass also represented in terms of temperature as,

$$
M=\frac{K T^{2}}{2} .
$$

It is clear that the $M, T$ and $S_{0}$ satisfy the first law of thermodynamics $\left(d M=T d S_{0}\right)$. Now, using the equations (6) and (7) one can obtain corrected entropy as (More (2005)),

$$
S=S_{0}-\frac{3}{2} \ln S_{0}-\frac{3}{16 S_{0}}+\cdots .
$$

By using the equation (15) one can write,

$$
S=K T-\frac{3}{2} \ln K T-\frac{3}{16 K T}+\cdots,
$$

where $K \equiv \pi^{2} l^{2}$ is a constant. In that case the first law of thermodynamics is valid only at $T=\frac{1}{8 K}$. Helmholtz free energy obtained via the following thermodynamics relation,

$$
F=-\int S d T
$$

which yields to the following corrected relation,

$$
F=-\frac{1}{2} K T^{2}+\frac{3}{2} T \ln K T-\frac{3}{2} T+\frac{3}{16} \frac{\ln T}{K} .
$$

Three last three terms of the above relation shows effect of higher order corrections. It is clear that the Helmholtz free energy is negative. There is a special temperature $T_{h}$ where correction terms have no effect on the Helmholtz free energy and obtained via the following equation,

$$
\frac{3}{2} T_{h} \ln K T_{h}-\frac{3}{2} T_{h}+\frac{3}{16} \frac{\ln T_{h}}{K}=0,
$$

So, for the $T \leq T_{h}$ correction terms increases value of the Helmholtz free energy, while for the $T \geq T_{h}$ correction terms decreases value of the Helmholtz free energy.

Specific heat is obtained using the following relation,

$$
C=T\left(\frac{d S}{d T}\right)
$$

which yields to the following corrected relation,

$$
C=K T-\frac{3}{2}+\frac{3}{16 K T}
$$

It is clear that the BTZ black hole is stable (specific heat is positive) without logarithmic corrections. However, effect of the logarithmic correction (leading-order correction) is presence of some instabilities at low temperature. Indeed, there is a critical temperature 
$T_{c}=\frac{3}{2 K}$ at leading-order so the black hole is in unstable phase at $T<T_{c}$ while is stable for $T \geq T_{c}$. However, in presence of higher order corrections, there are two critical temperatures $T_{c \pm}=\frac{3 \pm \sqrt{6}}{4 K}$, so the black hole is in stable phase for $T \leq T_{c-}$ and $T \geq T_{c+}$ while is in unstable phase at $T_{c-}<T<T_{c+}$. On the other hand, without logarithmic correction, effect of higher order correction is presence of a minimum in the specific heat as $C_{\min }=\frac{\sqrt{3}}{2}$ which happen at $T_{\min }=\frac{\sqrt{3}}{4 K}$.

We can see above statements in the Fig. 1. Solid line represent the ordinary case with $S=S_{0}$, while logarithmic correction case $\left(S=K T-\frac{3}{2} \ln K T\right)$ denoted by dashed blue line of the Fig. 1. Finally higher order corrected specific heat (using the equation (19) represented by dotted red line of the Fig. 1.

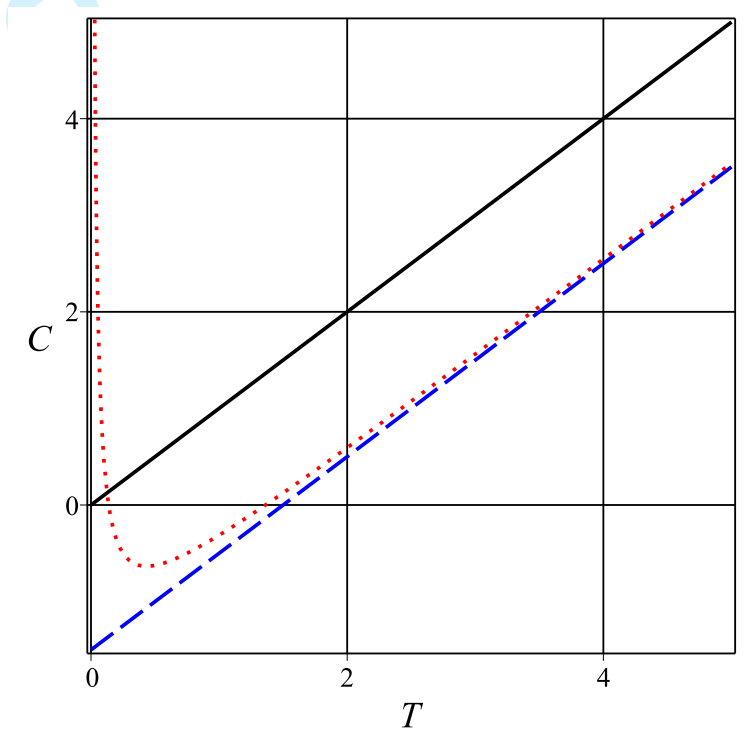

Figure 1: Specific heat in terms of temperature for the BTZ black hole. Solid line shows absence of correction. Dashed line shows logarithmic correction and dotted line shows higher order correction.

Now, by using the following thermodynamics relation,

$$
U=\int C d T
$$

one can obtain internal energy as follow,

$$
U=\frac{1}{2} K T^{2}-\frac{3}{2} T+\frac{3}{16} \frac{\ln T}{K}+c_{1},
$$

where $c_{1}$ is an integration constant which can be set via the following thermodynamics relation,

$$
S=\frac{U-F}{T}
$$


which tells that $c_{1}=-\frac{3}{16 K}$, hence,

$$
U=\frac{1}{2} K T^{2}-\frac{3}{2} T+\frac{3}{16 K}[-1+\ln T] .
$$

We find that the effect of higher order corrections is reduced initial value $(T=0)$ of internal energy. In presence of logarithmic correction, value of the internal energy is negative at low temperature, and there is a minima for the internal energy.

Finally, by using the black hole volume,

$$
V=\pi r_{+}^{2}
$$

which can be rewritten in terms of temperature as follow,

$$
V=\frac{4}{\pi} K^{2} T^{2}
$$

one can discuss about $P-V$ diagram which is illustrated by the Fig. 2. We can see no critical points in presence of and absence of correction terms hence there is no phase transition.

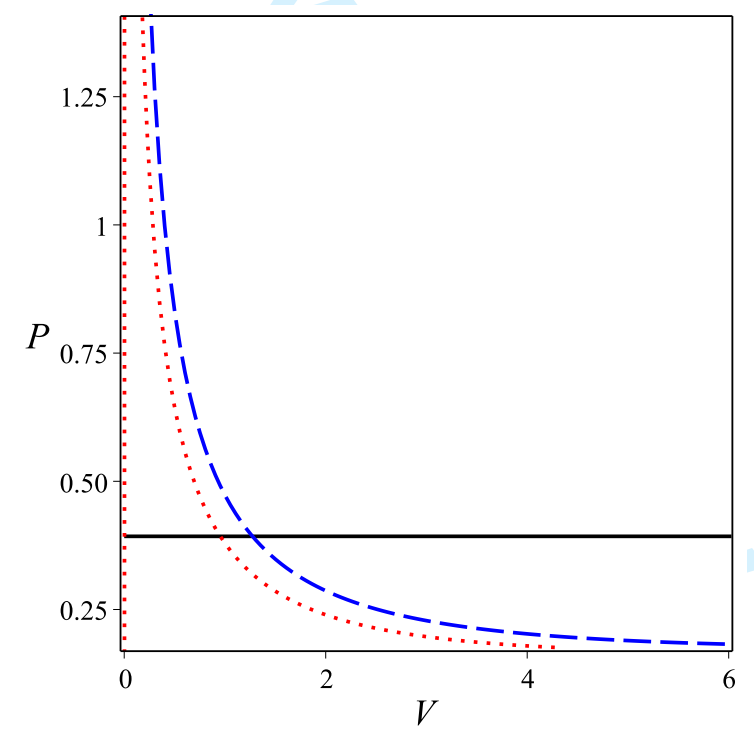

Figure 2: $P-V$ diagram of the BTZ black hole. Solid line shows absence of correction. Dashed line shows logarithmic correction and dotted line shows higher order correction. 


\section{AdS Schwarzschild black hole thermodynamics}

In this section we would like to consider a 4-dimensional AdS Schwarzschild black hole given by the following metric,

$$
\begin{aligned}
d s^{2}= & -\left(1-\frac{8 \pi M}{\Omega_{2} r}+\frac{r^{2}}{l^{2}}\right) d t^{2} \\
& +\left(1-\frac{8 \pi M}{\Omega_{2} r}+\frac{r^{2}}{l^{2}}\right)^{-1} d r^{2}+r^{2} d \Omega_{2}^{2}
\end{aligned}
$$

here, the parameter $l$ is related to the negative cosmological constant via $\Lambda=-\frac{3}{l^{2}}$. The Hawking temperature is given by,

$$
T=\frac{3 r_{+}^{2}+l^{2}}{4 \pi l^{2} r_{+}}
$$

where event horizon radius $r_{+}$is real positive root of the following equation,

$$
r^{3}+l^{2} r-\frac{8 \pi M l^{2}}{\Omega_{2}}=0,
$$

which is,

$$
\begin{aligned}
r_{+} & =\frac{1}{6}\left(108 A+12 \sqrt{12 l^{6}+81 A^{2}}\right)^{\frac{1}{3}} \\
& -\frac{2 l^{2}}{\left(108 A+12 \sqrt{12 l^{6}+81 A^{2}}\right)^{\frac{1}{3}}}
\end{aligned}
$$

where

$$
A=\frac{8 \pi M l^{2}}{\Omega_{2}}
$$

is a constant.

Also uncorrected entropy of a 4-dimensional AdS Schwarzschild is given by,

$$
S_{0}=\frac{\Omega_{2} r_{+}^{2}}{4}
$$

Hence, one can obtain relation between temperature and entropy as,

$$
T=\frac{12 S_{0}+l^{2} \Omega_{2}}{8 \pi l^{2} \sqrt{\Omega_{2}} \sqrt{S_{0}}}
$$

or inverse,

$$
S_{0}=\frac{\Omega_{2} l^{2}}{9}\left[2 \pi^{2} l^{2} T^{2}+\pi l T \sqrt{4 \pi^{2} l^{2} T^{2}-3}-\frac{3}{4}\right] .
$$

Now, one can obtain corrected entropy as [29],

$$
S=S_{0}-\ln \frac{1}{n}-\ln S_{0}-\frac{5}{36 S_{0}}+\cdots
$$


In the case of $n=1$ the second term of r.h.s vanishes and we have,

$$
S=S_{0}-\ln S_{0}-\frac{5}{36 S_{0}}
$$

where $S_{0}$ given by the equation (38).

By using the equation (20) one can obtain corrected Helmholtz free energy as follow,

$$
\begin{aligned}
F & =-\frac{\Omega_{2} l\left(4 \pi^{2} l^{2} T^{2}-3\right)^{\frac{3}{2}}}{108 \pi} \\
& -\frac{2 \pi^{2} l^{4} \Omega_{2}}{27} T^{3}+\frac{l^{2} \Omega_{2}}{12} T-2 T \ln 6 \\
& +T \ln \left[\Omega_{2} l^{2}\left(8 \pi^{2} l^{2} T^{2}+4 \pi l T \sqrt{4 \pi^{2} l^{2} T^{2}-3}-3\right)\right] \\
& -\frac{\sqrt{4 \pi^{2} l^{2} T^{2}-3}}{l \pi}+\frac{40 \pi^{2}}{27 \Omega_{2}} T^{3} \\
& -\frac{5 T}{3 l^{2} \Omega_{2}}-\frac{5\left(4 \pi^{2} l^{2} T^{2}-3\right)^{\frac{3}{2}}}{27 \pi \Omega_{2} l^{3}}
\end{aligned}
$$

Three first terms of r.h.s are corresponding to uncorrected Helmholtz free energy, while three last terms are consequences of higher order corrections. Also three middle terms are corresponding to the logarithmic corrections. We find that all corrections have infinitesimal contributions in the Helmholtz free energy and don't change general behavior of the Helmholtz free energy.

Now, we can use the equation (23) to evaluate corrected specific heat,

$$
\begin{aligned}
C & =\frac{l^{2}}{12}\left(B_{1}+B_{2} T\right) T \\
& -\frac{\left(B_{1}+B_{2} T\right) T}{B_{1} T-\Omega_{2}} \\
& +\frac{5\left(B_{1}+B_{2} T\right) T}{3 l^{2}\left(B_{1} T-\Omega_{2}\right)^{2}},
\end{aligned}
$$

where we defined,

$$
B_{1}=4 \pi \Omega_{2} l\left(\frac{2}{3} \pi l T+\frac{\sqrt{4 \pi^{2} l^{2} T^{2}-3}}{3}\right)
$$

and

$$
B_{2}=4 \pi \Omega_{2} l\left(\frac{2}{3} \pi l+\frac{4 \pi^{2} l^{2} T}{\sqrt{4 \pi^{2} l^{2} T^{2}-3}}\right) .
$$

The second line of the equation (42) is effect of the logarithmic correction while the last line show higher order correction. It is clear that the 4-dimensional AdS Schwarzschild black hole without logarithmic correction is completely stable, while effect of leading-order correction is presence of some unstable regions. If higher order corrections neglected, then we can see unstable/stable black hole phase transition. These are illustrated by the Fig. 3. We can see 


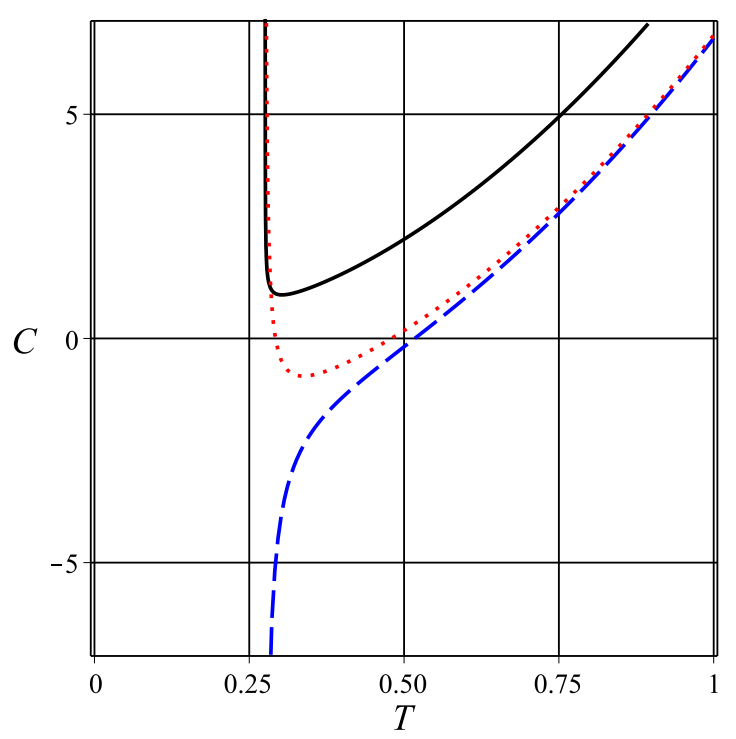

Figure 3: Specific heat in terms of temperature for the AdS black hole. Solid line shows absence of correction. Dashed line shows logarithmic correction and dotted line shows higher order correction.

asymptotic behavior (dashed blue line of the Fig. 3) which may be cause of phase transition and will be clear when we discuss about critical points.

Similar to the previous section by using the relation (29) and,

$$
P=-\frac{d F}{d V}
$$

one can obtain $P-V$ diagram which represented by the Fig. 4 . We can see, in presence of correction terms, there is some points where $\frac{d P}{d V}=0$, hence it may be possible to find some critical points and phase transition.

Finally, we can study internal energy using the equation (25) and find that effect of corrections of entropy is infinitesimal and negligible.

\section{Conclusion}

In this paper, we considered higher order corrections of entropy for the BTZ and AdS Schwarzschild black holes and studied thermodynamics of mentioned black holes. First of all we considered non-rotating BTZ black hole and obtained relation between Hawking temperature and original entropy of black hole. Then, wrote higher order corrected entropy and evaluated the Helmholtz free energy to obtain temperature dependence corrected terms. Then, we calculated specific heat and found that leading-order corrections yields to the black 


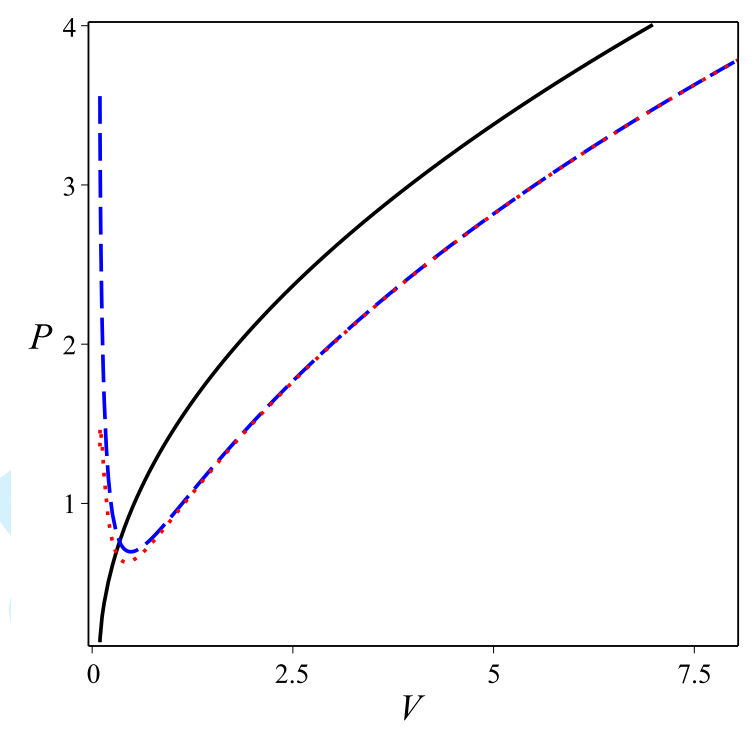

Figure 4: $P-V$ diagram of the AdS black hole. Solid line shows absence of correction. Dashed line shows logarithmic correction and dotted line shows higher order correction.

hole instability, while higher order corrections help to remove instabilities. In summary, we found that when size of black hole reduced due to the Hawking radiation ant thermal fluctuation be important, the black hole translate to the unstable phase. Finally we obtained internal energy of BTZ black hole and have shown infinitesimal effects of higher order corrections. In this case we found that there are no critical points and phase transition.

In the second sector of this paper, we considered AdS Schwarzschild black hole in four dimensions and investigate effects of higher order corrections of the entropy on the thermodynamics quantities. We obtained corrected Helmholtz free energy and internal energy of black holes and found that effects of higher order corrections are negligible. But we have shown that effects of higher order correction on the specific heat are important and may yield to some instabilities. Hence, we have shown unstable to stable black hole phase transition in presence of logarithmic correction. It means that there are critical points corresponding to the entropy corrected black holes. For the future works it is possible to find holographic dual of the black holes which may be a Van der waals fluid which accessible in the Lab to test quantum gravity effects. It may be also interesting to use astrophysical data of some black holes to fit correction coeficients of the entropy.

\section{Acknowledgements}

The Financial Support of the Research Council of Damghan University with the Grant Number 96/phys/129/287 is Acknowledged. 


\section{References}

[1] J. D. Bekenstein, Phys. Rev. D7 (1973) 2333

[2] L. Susskind, J. Math. Phys. 36 (1995) 6377

[3] R. Bousso, Rev. Mod. Phys. 74 (2002) 825

[4] A. Strominger, C. Vafa, Phys. Lett. B379 (1996) 99

[5] A. Ashtekar, J. Baez, A. Corichi, K. Krasnov, Phys. Rev. Lett. 80 (1998) 905

[6] S. Carlip, Phys. Rev. Lett. 82 (1999) 2828

[7] R. Kaul, P. Majumdar, Phys. Lett. B439 (1998) 267

[8] S. Das, R. K. Kaul and P. Majumdar, Phys. Rev. D 63 (2001) 044019

[9] S. N. Solodukhin, Phys. Rev. D57 (1998) 2410

[10] A. Chatterjee, P. Majumdar, Phys. Rev. Lett. 92 (2004) 141301

[11] D. Bak and S. J. Rey, Class. Quant. Grav. 17 (2000) L1

[12] S. K. Rama, Phys. Lett. B 457 (1999) 268

[13] S. Das, P. Majumdar, R.K. Bhaduri, Class. Quantum Gravity 19 (2002) 2355

[14] J. Sadeghi, B. Pourhassan, F. Pourasadollah, Eur. Phys. J. C74 (2014) 2793

[15] J. Sadeghi, B. Pourhassan, H. Farahani, Commun. Theor. Phys. 62 (2014) 358

[16] W. Xu and L. Zhao, Phys. Rev. D 87 (2013) 124008

[17] B. Pourhassan, Mod. Phys. Lett. A31 (2016) 1650057

[18] J. Sadeghi, B. Pourhassan, F. Rahimi, Can. J. Phys. 92 (2014) 1638

[19] A. Pourdarvish, J. Sadeghi, H. Farahani, B. Pourhassan, Int. J. Theor. Phys. 52 (2013) 3560

[20] B. Pourhassan, M. Faizal, Europhys. Lett. 111 (2015) 40006

[21] M. Faizal, B. Pourhassan, Phys. Lett. B 751 (2015) 487

[22] B. Pourhassan, M. Faizal, Phys. Lett. B755 (2016) 444

[23] B. Pourhassan, M. Faizal, U. Debnath, Eur. Phys. J. C 76 (2016) 145

[24] J. Sadeghi, B. Pourhassan, M. Rostami, Phys. Rev. D 94 (2016) 064006 
[25] S. Upadhyay, B. Pourhassan, H. Farahani, Phys. Rev. D 95 (2017) 106014

[26] B. Pourhassan, M. Faizal, Nuclear Physics B 913 (2016) 834

[27] B. Pourhassan, M. Faizal, Eur. Phys. J. C 77 (2017) 96

[28] B. Pourhassan, M. Faizal, S. Capozziello, Annals of Physics 377 (2017) 108

[29] S.S. More, Class. Quant. Grav. 22 (2005) 4129

[30] M. Banados, C. Teitelboim, J. Zanelli, Phys. Rev. Lett. 69 (1992) 1849

[31] J. Sadeghi, H. Farahani, B. Pourhassan and M. Noorbakhsh, Physics Letters B 703 (2011) 14 